\title{
Malnutrición hospitalaria: etiología y criterios para su diagnóstico y clasificación
}

\author{
Hospital malnutrition: etiology and criteria for diagnosis and classification \\ Desnutrição hospitalar: etiologia e critérios para diagnóstico e classificação
}

\author{
Eduardo Lobatón ${ }^{1 *}$
}

Recibido: 19 de junio de 2019. Aceptado para publicación: 2 de septiembre de 2019.

Publicado en línea: 9 de noviembre de 2019

https://doi.org/10.35454/rncm.v3n1.019

\section{Resumen}

La malnutrición hospitalaria (desnutrición) es de alta prevalencia en el mundo, reportándose hasta $30 \%-50 \%$. A diferencia de la observada en salud pública, esta se caracteriza por la activación e instalación de la respuesta inflamatoria de manera aguda o crónica, y una atenuada respuesta al soporte nutricional. Es por ello que parámetros tradicionales para el diagnóstico de malnutrición en salud pública como la albúmina han fracasado en su aplicación en el área clínica y obligado a replantear los criterios usados para el diagnóstico de malnutrición hospitalaria desde esta nueva perspectiva. Tanto la Sociedad Americana de Nutrición Enteral y Parenteral (ASPEN, por sus siglas en inglés), como la Academia Americana de Nutrición y Dietética (AND, por sus siglas en inglés) y posteriormente la Sociedad Europea de Nutrición Clínica y Metabolismo (ESPEN, por sus siglas en inglés) han emitido consensos para el diagnóstico y clasificación de la malnutrición hospitalaria. Recientemente la iniciativa de Liderazgo Global de Malnutrición (GLIM, siglas en inglés), tomando como base estos consensos, ha planteado una nueva clasificación desde un enfoque más global. El presente artículo revisa varios conceptos alrededor de este tema.

\section{Summary}

Hospital malnutrition (undernutrition) is of high prevalence in the world having reported up to $30-50 \%$. Unlike the one observed in public health, this is characterized by the activation and installation of the inflammatory response in an acute or chronic manner, and an attenuated response to nutritional support. That is why traditional parameters for the diagnosis of malnutrition in public health such as albumin have failed in its application in the clinical area and forced to rethink the criteria used for the diagnosis of hospital malnutrition from this new perspective. Both the American Society for Parenteral and Enteral Nutrition (ASPEN) as well as the American Academy of Nutrition and Dietetics (AND) and subsequently the European Society for Clinical Nutrition and Metabolism (ESPEN, for its acronym in English) have issued consensus for the diagnosis and classification of hospital malnutrition. Recently, the global malnutrition leadership initiative (GLIM), based on these consensus, has proposed a new classification from a more global approach. This article reviews this and other concepts around this topic.

Keywords: Hospital malnutrition; Inflammatory response; Cachexia; GLIM.

\section{Resumo}

A desnutrição hospitalar é de alta prevalência no mundo, relatando até $30 \%$ - 50\%. Diferentemente do observado em saúde pública, esta é caracterizada pela ativação e instalação da resposta inflamatória de maneia aguda ou crónica e uma resposta atenuada ao suporte nutricional. Por isso, os parâmetros tradicionais para o diagnóstico de desnutrição em saúde pública, como a albumina, falharam em sua aplicação na área clínica e forçaram a repensar os critérios utilizados para o diagnóstico de desnutrição hospitalar sob nova perspetiva. Tanto a Sociedade Americana de Nutrição Enteral e Parenteral (ASPEN, por em inglês), como a Academia Americana de Nutrição e Dietética (AND) e, posteriormente, a Sociedade Europeia de Nutrição Clínica e Metabolismo (ESPEN, por em inglês) emitiram consensos para o diagnóstico e classificação da desnutrição hospitalar. Recentemente, a iniciativa global de liderança em desnutrição (GLIM), tomando como base estes consensos, propôs uma nova classificação a partir de uma abordagem mais global. Este artigo analisa vários conceitos sobre esse tópico.

Palavras-chave: desnutrição hospitalar, resposta inflamatória, caquexia, GLIM.

Palabras clave: malnutrición hospitalaria, respuesta inflamatoria, caquexia, GLIM.

Dignity Health Bakersfield Memorial Hospital, California, Estados Unidos. 


\section{INTRODUCCIÓN}

La malnutrición es la condición que resulta de un desbalance en la dieta en la cual ciertos nutrientes pueden estar en menor o mayor proporción o en las proporciones inadecuadas. Dependiendo de si estos están en déficit o exceso esta se puede dar en la forma de desnutrición o sobrealimentación. En salud pública la malnutrición en países en desarrollo es prevalente en la forma de desnutrición como resultado de la falta de alimentos debido a extrema pobreza, hambrunas, desastres naturales y conflictos, este tipo de malnutrición puede darse por un déficit calórico (tipo marasmo), un déficit proteico (tipo kwashiorkor) o una combinación de ambos ${ }^{(1)}$. Sin embargo, desde hace varios años la coexistencia del sobrepeso y la obesidad genera una doble carga de malnutrición en estos países ${ }^{(2)}$. En países desarrollados prevalece la malnutrición por exceso.

En el ámbito hospitalario la malnutrición está presente principalmente en la forma de desnutrición asociada a la enfermedad o a la injuria ${ }^{(3)}$, con una prevalencia de alrededor de $30 \%$ a $50 \%{ }^{(4)}$.

A diferencia de la desnutrición observada en salud pública, en el área clínica su etiología está relacionada con la alteración de los requerimientos nutricionales debido a la respuesta inflamatoria la cual induce a anorexia y disminución en la ingesta de alimentos, pobre absorción de nutrientes o excesiva pérdida de los mismos, a alteraciones metabólicas con aumento del gasto energético y del catabolismo proteico muscular ${ }^{(5)}$. Su presencia está asociada a un incremento de las comorbilidades principalmente infecciosas, pérdida de masa muscular, pobre cicatrización de heridas, alteración en la calidad de vida, mayor estancia hospitalaria, costos y mortalidad $^{(6-9)}$.

Si bien la desnutrición observada en el contexto de salud pública responde fácilmente si la causa (falta de alimentos) es revertida, la observada en el área clínica no responde en la misma proporción. Un concepto que ha quedado en desuso es el del balance energético, a través del cual el aporte debería ser igual al gasto calórico. Siguiendo este simple principio, en la década de los 70 y 80 era popular el uso de hasta $40 \%$ - $50 \%$ calorías $/ \mathrm{kg}$ de peso a fin de compensar el aumento en los requerimientos calóricos debido a la enfermedad. Esto más que revertir la desnutrición trajo consigo muchas complicaciones asociadas a la hiperalimentación como hígado graso, esteatosis, hiperglicemia, hipercapnia, entre otros ${ }^{(10,11)}$. De la misma manera ha sido demostrado que la administración de más de $2 \mathrm{~g}$ de proteína por $\mathrm{kg}$ de peso no tiene mayor impacto sobre la mortalidad y calidad de vida del paciente ${ }^{(12,13)}$. Además, el balance nitrogenado negativo observado en pacientes en estado crítico solo es atenuado más no revertido a pesar de un suficiente aporte calórico y proteico ${ }^{(14)}$. Sin duda, parte de la solución va más allá de conseguir un balance positivo de nutrientes.

\section{MALNUTRICIÓN HOSPITALARIA Y RESPUESTA INFLAMATORIA}

La caquexia es el término frecuentemente empleado para referirse a aquellos pacientes con avanzado grado de desnutrición. Este síndrome es prevalente en pacientes con enfermedades crónicas tales como SIDA, cáncer, insuficiencia renal, insuficiencia hepática, enfermedad pulmonar obstructiva crónica entre otros. ESPEN (Sociedad Europea de Nutrición Clínica y Metabolismo) define la caquexia como un síndrome multifactorial caracterizado por severa pérdida de peso, y masa muscular con o sin pérdida de masa grasa, así como un incremento en el catabolismo proteico vinculado a la enfermedad subyacente (según ESPEN, caquexia es sinónimo de malnutrición relacionada con enfermedad crónica en presencia de inflamación) $)^{(15,16)}$. El síndrome caquéctico tiene dos características fundamentales. Por un lado, una marcada falta de apetito, $y$, por otro, alteraciones metabólicas que incluyen aumento del catabolismo proteico y alteración del metabolismo de carbohidratos y lípidos ${ }^{(17)}$. En su origen, la caquexia está vinculada a la activación del sistema inmune debido a la presencia de enfermedad. Una vez el sistema inmunológico es activado se desencadena una serie de eventos conocidos todos ellos, en conjunto, como respuesta inflamatoria. Esta respuesta es regulada a través de las citoquinas, proteínas que regulan la función celular y la comunicación intercelular. Estas proteínas, de manera experimental, son capaces de reproducir en el laboratorio los signos y síntomas observados en pacientes caquécticos. Entre las citoquinas más conocidas se encuentran el Factor de Necrosis Tumoral alfa, conocida inicialmente con el nombre de "caquectina" por su poder de suprimir el apetito y alterar el metabolismo como se observa en la caquexia ${ }^{(17)}$. Si bien esta es una respuesta biológica fisiológica que permite al organismo hacer frente a la agresión, reparar tejidos dañados y cicatrizar heridas, la instalación crónica termina consumiendo las reservas del huésped y generando caquexia ${ }^{(15,16,18)}$.

Es en este contexto donde indicadores bioquímicos clásicos de desnutrición como la albúmina y prealbú- 
mina (también llamada transtirretina) pierden valor ya que son afectados de diferentes maneras durante la respuesta inflamatoria ${ }^{(19)}$. En el caso de la albúmina la síntesis disminuye en el hígado para dar lugar a la producción de proteínas de fase aguda tal como la proteína $\mathrm{C}$ reactiva (cuya función es la de activar el sistema del complemento a fin de facilitar la fagocitosis y la apoptosis), o permitiendo su fuga fuera del comportamiento vascular en el edema ${ }^{(20,21)}$. Sin embargo, en la práctica, niveles de albúmina o prealbúmina bajos siguen siendo atribuidos a un déficit proteico, lo cual no deja de ser una razón válida, pero en presencia de enfermedad la respuesta inflamatoria es la principal causa. Además, el estado de hidratación puede confundir aún más los valores como en el caso de falla hepática o renal ${ }^{(22,23)}$. Es por ello que estos indicadores han fracasado en su especificidad y en su sensibilidad como indicadores de estado nutricional, pero se correlacionan muy bien como indicadores de pronóstico, de morbi-mortalidad y de inflamación al igual que la proteína $\mathrm{C}$ reactiva ${ }^{(20,24)}$. Por lo tanto, mientras en salud pública la desnutrición está vinculada a la falta de alimentos, en el hospital, salvo algunas excepciones (como la anorexia nerviosa), está ligada a la respuesta inflamatoria producto de la enfermedad subyacente.

\section{DIAGNÓSTICO Y CLASIFICACIÓN SEGÚN LOS CRITERIOS ASPEN/AND}

En el año 2012, según este enfoque la AND y ASPEN emitieron un consenso para la identificación y docu- mentación de malnutrición adulta hospitalaria (desnutrición) donde se considera la presencia de inflamación en su etiología (Figura 1) ${ }^{(25,26)}$. En este consenso, se replantea el uso de los indicadores nutricionales proponiendo 6, donde la presencia de 2 o más es suficiente para diagnosticar malnutrición (desnutrición). Se propone la cuantificación del grado de severidad según la presencia o ausencia de inflamación (Tabla 1).

Además, plantea que la evaluación nutricional debería mantener este mismo enfoque al momento de su realización. Se emiten algunas recomendaciones, por ejemplo:

- La historia y el diagnóstico clínico son útiles para evaluar el riesgo de malnutrición, pero a su vez para evaluar la presencia o no de inflamación según el tipo de enfermedad, como se aprecia en la Figura 1.

- El examen físico enfocado en la evaluación del estado nutricional puede mostrar la presencia de varias características clínicas de malnutrición como son la pérdida de peso, retención de fluidos, pérdida de masa grasa o muscular, mientras que los signos clínicos tales como fiebre o hipotermia, taquicardia, hiperventilación e hiperglicemia la presencia de respuesta inflamatoria.

- Indicadores antropométricos tales como el IMC en valores extremos reflejan un pobre estado nutricional, aunque la malnutrición puede ocurrir en cualquier valor de IMC.

- Valores de albúmina y prealbúmina deberían ser interpretados con cautela y más bien deberían ser usados

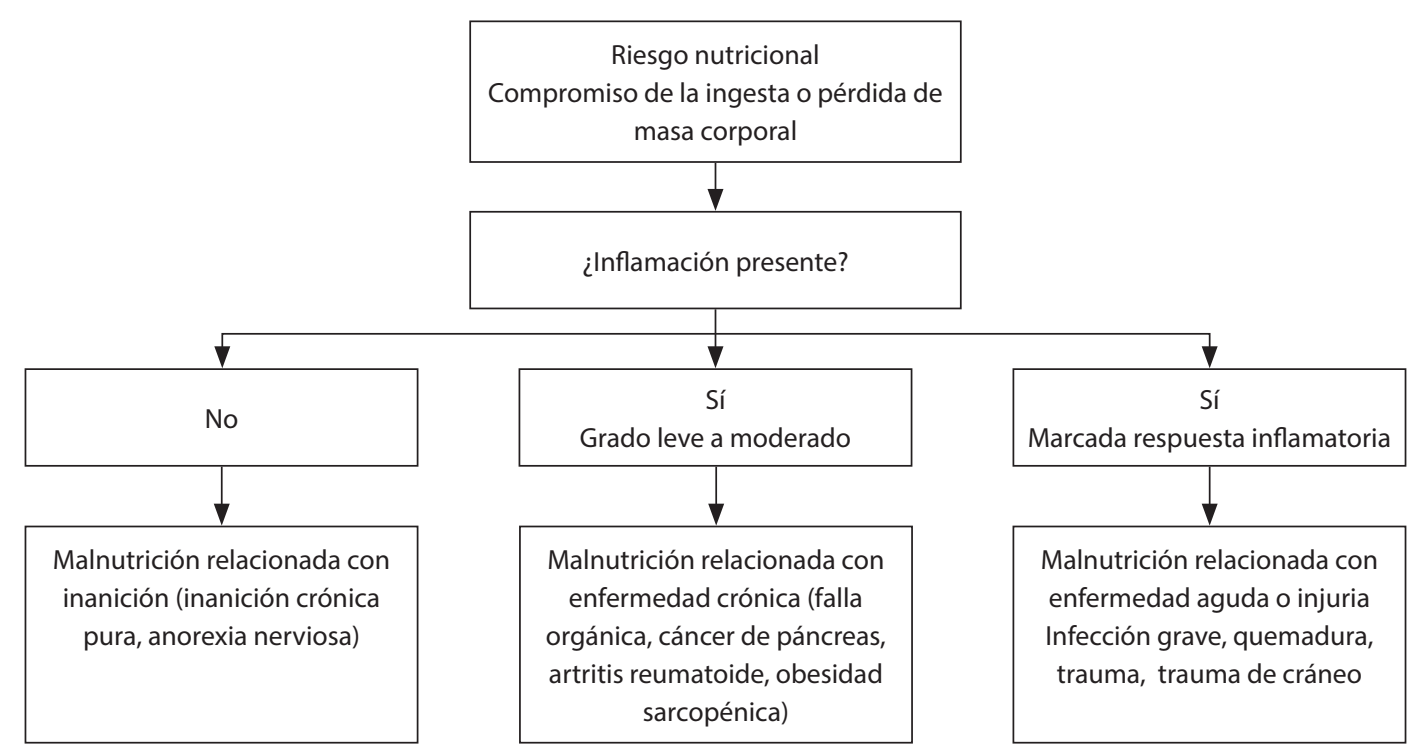

Figura 1. Tipos de malnutrición según criterios etiológicos. Traducido y publicado con permiso de White et al(25). 
Tabla 1. Características clínicas para establecer un diagnóstico de malnutrición según AND / ASPEN. Traducido y publicado con permiso de White et $\mathbf{a l}^{(25)}$

\begin{tabular}{|c|c|c|c|c|c|c|c|c|c|c|c|c|}
\hline \multirow[b]{2}{*}{$\begin{array}{l}\text { Características } \\
\text { clínicas }\end{array}$} & \multicolumn{4}{|c|}{$\begin{array}{l}\text { Malnutrición en el contexto de } \\
\text { enfermedad aguda o injuria }\end{array}$} & \multicolumn{4}{|c|}{$\begin{array}{l}\text { Malnutrición en el contexto de } \\
\text { enfermedad crónica }\end{array}$} & \multicolumn{4}{|c|}{$\begin{array}{c}\text { Malnutrición en el contexto } \\
\text { de circunstancias sociales o } \\
\text { ambientales }\end{array}$} \\
\hline & \multicolumn{2}{|c|}{$\begin{array}{l}\text { Malnutrición } \\
\text { No severa } \\
\text { (moderada) }\end{array}$} & \multicolumn{2}{|c|}{$\begin{array}{c}\text { Malnutrición } \\
\text { severa }\end{array}$} & \multicolumn{2}{|c|}{$\begin{array}{l}\text { Malnutrición } \\
\text { No severa } \\
\text { (moderada) }\end{array}$} & \multicolumn{2}{|c|}{$\begin{array}{l}\text { Malnutrición } \\
\text { severa }\end{array}$} & \multicolumn{2}{|c|}{$\begin{array}{l}\text { Malnutrición } \\
\text { No severa } \\
\text { (moderada) }\end{array}$} & \multicolumn{2}{|c|}{$\begin{array}{l}\text { Malnutrición } \\
\text { severa }\end{array}$} \\
\hline $\begin{array}{l}\text { Ingesta de } \\
\text { energía }\end{array}$ & \multicolumn{2}{|c|}{$\begin{array}{l}<75 \% \\
\text { requerimientos } \\
\text { energéticos }> \\
7 \text { días }\end{array}$} & \multicolumn{2}{|c|}{$\begin{array}{l}\leq 50 \% \\
\text { requerimientos } \\
\text { energéticos } \geq \\
5 \text { días }\end{array}$} & \multicolumn{2}{|c|}{$\begin{array}{l}<75 \% \\
\text { requerimientos } \\
\text { energéticos por } \\
\geq 1 \text { mes }\end{array}$} & \multicolumn{2}{|c|}{$\begin{array}{c}\leq 75 \% \\
\text { requerimientos } \\
\text { energéticos por } \geq \\
1 \text { mes }\end{array}$} & \multicolumn{2}{|c|}{$\begin{array}{l}\quad<75 \% \\
\text { requerimientos } \\
\text { energéticos por } \\
\quad \geq 3 \text { meses }\end{array}$} & \multicolumn{2}{|c|}{$\begin{array}{l}\leq 50 \% \\
\text { requerimientos } \\
\text { energéticos por } \geq \\
1 \text { mes }\end{array}$} \\
\hline $\begin{array}{l}\text { Pérdida de } \\
\text { peso }\end{array}$ & $\begin{array}{c}\% \\
1-2 \\
5 \\
7,5\end{array}$ & $\begin{array}{l}\text { Tiempo } \\
1 \text { sem } \\
1 \text { mes } \\
3 \text { mes }\end{array}$ & $\begin{array}{c}\% \\
>2 \\
>5 \\
>7,5\end{array}$ & $\begin{array}{l}\text { Tiempo } \\
1 \text { sem } \\
1 \text { mes } \\
3 \text { mes }\end{array}$ & $\begin{array}{c}\% \\
5 \\
7,5 \\
10 \\
20\end{array}$ & $\begin{array}{c}\text { Tiempo } \\
1 \text { mes } \\
3 \text { mes } \\
6 \text { mes } \\
1 \text { año }\end{array}$ & $\begin{array}{c}\% \\
>5 \\
>7,5 \\
>10 \\
>20\end{array}$ & $\begin{array}{c}\text { Tiempo } \\
1 \text { mes } \\
3 \text { mes } \\
6 \text { mes } \\
1 \text { año }\end{array}$ & $\begin{array}{c}\% \\
5 \\
7,5 \\
10 \\
20\end{array}$ & $\begin{array}{c}\text { Tiempo } \\
1 \text { mes } \\
3 \text { mes } \\
6 \text { mes } \\
1 \text { año }\end{array}$ & $\begin{array}{c}\% \\
>5 \\
>7,5 \\
>10 \\
>20\end{array}$ & $\begin{array}{c}\text { Tiempo } \\
1 \text { mes } \\
3 \text { mes } \\
6 \text { mes } \\
1 \text { año }\end{array}$ \\
\hline Masa grasa & \multicolumn{2}{|c|}{ Leve } & \multicolumn{2}{|c|}{ Moderada } & \multicolumn{2}{|c|}{ Leve } & \multicolumn{2}{|c|}{ Severa } & \multicolumn{2}{|r|}{ Leve } & \multicolumn{2}{|c|}{ Severa } \\
\hline Masa muscular & \multicolumn{2}{|c|}{ Leve } & \multicolumn{2}{|c|}{ Moderada } & \multicolumn{2}{|c|}{ Leve } & \multicolumn{2}{|c|}{ Severa } & \multicolumn{2}{|c|}{ Leve } & \multicolumn{2}{|c|}{ Severa } \\
\hline $\begin{array}{l}\text { Acumulación } \\
\text { de líquidos }\end{array}$ & \multicolumn{2}{|c|}{ Leve } & \multicolumn{2}{|c|}{$\begin{array}{c}\text { Moderada a } \\
\text { severa }\end{array}$} & \multicolumn{2}{|c|}{ Leve } & \multicolumn{2}{|c|}{ Severa } & & Leve & \multicolumn{2}{|c|}{ Severa } \\
\hline $\begin{array}{l}\text { Fuerza del } \\
\text { puño }\end{array}$ & \multicolumn{2}{|c|}{ No aplica } & \multicolumn{2}{|c|}{ Reducido } & \multicolumn{2}{|c|}{ No aplica } & \multicolumn{2}{|c|}{ Reducido } & \multicolumn{2}{|c|}{ No aplica } & \multicolumn{2}{|c|}{ Reducido } \\
\hline
\end{tabular}

como indicador del pronóstico del paciente y evaluar el grado de inflamación junto con los valores de proteina $\mathrm{C}$ reactiva, leucocitos y glucosa en sangre.

- Un balance nitrogenado negativo, así como un incremento de la tasa metabólica basal también pueden ser usados para estimar la presencia de respuesta inflamatoria sistémica.

- La información de la historia de la ingesta antes de la admisión, el empleo de un recordatorio de 24 horas o un conteo calórico pueden ser usados para evidenciar una inadecuada ingesta de nutrientes.

- La fuerza de agarre o del puño puede ser usada para documentar una declinación en la capacidad funcional.

\section{DIAGNÓSTICO Y CLASIFICACIÓN SEGÚN LOS CRITERIOS ESPEN}

En el año 2017, ESPEN establece criterios para el diagnóstico de malnutrición hospitalaria similares a los de la ADN y ASPEN (Figura 2), pero haciendo énfasis en el rol de la fisiopatología, distinguiendo entre la malnutrición relacionada con enfermedad, con o sin inflamación, y malnutrición sin enfermedad. Esta cla- sificación incluye a la caquexia por cáncer y otros tipos de caquexia en la categoría de malnutrición relacionada con enfermedad crónica en presencia de inflamación. Los desórdenes neurológicos (como el accidente cerebrovascular, el Parkinson, esclerosis lateral amiotrófica y demencia, entre otros), problemas psiquiátricos (anorexia nerviosa, depresión) y problemas de malabsorción (enfermedad celiaca, síndrome de intestino corto) los ubica en la categoría de malnutrición relacionada con enfermedad en ausencia de inflamación y considera la falta de alimentos, problemas socioeconómicos y sicológicos como las causas de la malnutrición en ausencia de enfermedad ${ }^{(27)}$.

\section{DIAGNÓSTICO Y CLASIFICACIÓN SEGÚN LOS CRITERIOS GLIM}

Recientemente la iniciativa "Liderazgo Global de Malnutrición" (GLIM) con la intención de tener un enfoque más global y práctico para el diagnóstico y clasificación de la malnutrición hospitalaria definió criterios nutricionales de dos tipos: criterios fenotípicos (pérdida involuntaria de peso, bajo índice de masa 


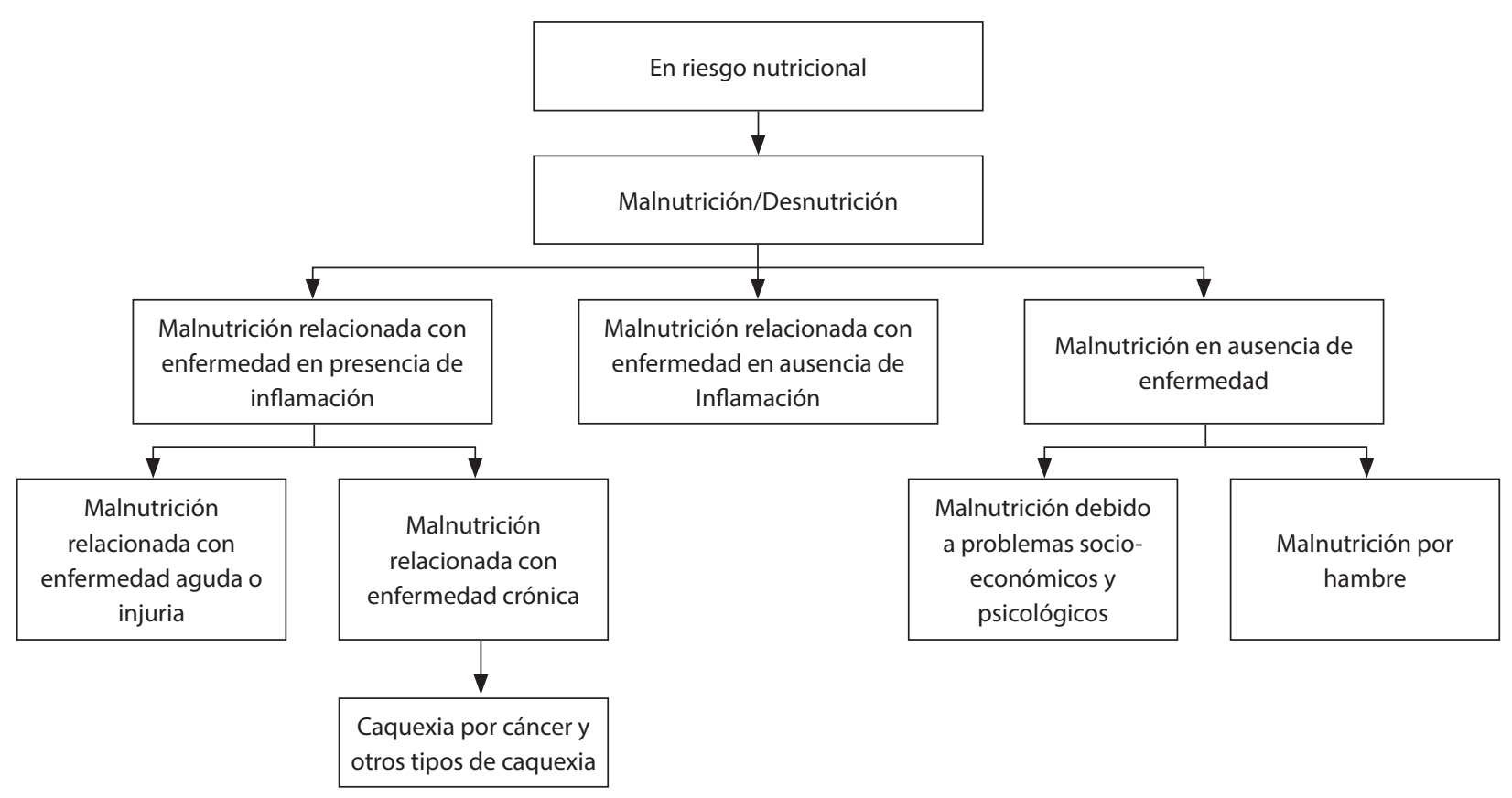

Figura 2. Diagnósticos de malnutrición, desde riesgo nutricional y una simple definición de malnutrición hasta uno basado en su etiología(27).

corporal [IMC], reducción de masa magra) y criterios etiológicos (presencia de inflamación, reducción de la ingesta) ${ }^{(28)}$. Se tomaron como punto de partida el consenso de la AND y ASPEN, y los criterios de la ESPEN (Tabla 2). Según este consenso global, se requiere al menos un criterio fenotípico y otro etiológico para establecer el diagnóstico de malnutrición. El grado de severidad (moderada o severa) se establece de acuerdo con los criterios fenotípicos (Tabla 3).

A diferencia del consenso de la AND y ASPEN, el consenso GLIM incorpora como uno de los indicadores nutricionales un bajo IMC y por otro lado ya no es necesaria la medición de la fuerza de agarre o puño a menos que sea imposible evaluar la masa muscular con métodos de valoración de composición corporal o antropométricos.

Es importante mencionar que la medición de la fuerza de agarre o del puño es un buen indicador de la fuerza muscular corporal total y se utiliza para diagnosticar la sarcopenia y el síndrome de fragilidad, y no solo correlaciona con fuerza si no también con masa muscular $^{(29-31)}$. La fuerza de agarre ha sido empleada para diferenciar los individuos que teniendo un bajo IMC están realmente malnutridos de aquellos que no ${ }^{(32)}$, por

Tabla 2. Criterios GLIM fenotípicos y etiológicos para el diagnóstico de malnutrición hospitalaria

\begin{tabular}{|l|l|l|l|}
\hline \multicolumn{2}{|c|}{ Criterio fenotípico } & & \multicolumn{2}{c|}{ Criterio etiológico } \\
\hline Pérdida de peso \% & $\begin{array}{l}>5 \% \text { en los pasados } 6 \text { meses o } \\
>10 \% \text { en más de } 6 \text { meses }\end{array}$ & $\begin{array}{l}\text { Ingesta de alimentos } \\
\text { reducida o disminución } \\
\text { en su asimilación }\end{array}$ & $\begin{array}{l}\text { Consumo de } 50 \% \text { de los requerimientos } \\
>1 \text { semana, o cualquier reducción por } \\
\text { más de 2 semanas, o cualquier afección } \\
\text { gastrointestinal que afecte la asimilación de } \\
\text { los alimentos }\end{array}$ \\
\hline $\begin{array}{l}\text { Bajo Índice de Masa } \\
\text { Corporal (IMC kg/m }\end{array}$ & $\begin{array}{l}<18,5 \mathrm{si}<70 \text { años o } \\
<22 \mathrm{si}>70 \text { años }\end{array}$ & Inflamación & $\begin{array}{l}\text { Enfermedad aguda/trauma o enfermedad } \\
\text { crónica }\end{array}$ \\
\hline Masa muscular reducida & Medido por método confiable & & \\
\hline
\end{tabular}


Tabla 3. Criterios fenotípicos para el diagnóstico de la severidad de la malnutrición ${ }^{(28)}$

\begin{tabular}{|l|l|l|l|}
\hline \multirow{2}{*}{} & \multicolumn{2}{|c|}{ Criterio fenotípico } \\
\cline { 2 - 4 } & \multicolumn{1}{|c|}{$\%$ de pérdida de peso } & Bajo IMC (kg/m²) & Reducción de masa muscular \\
\hline $\begin{array}{l}\text { Grado } 1 \text { Malnutrición Moderada } \\
\text { (Requiere un criterio fenotípico que } \\
\text { cumpla este grado) }\end{array}$ & $\begin{array}{l}5 \%-10 \% \text { en los últimos } 6 \text { meses, } \\
10 \%-20 \% \text { arriba de } 6 \text { meses }\end{array}$ & $\begin{array}{l}<20 \mathrm{si}<70 \text { años o }<22 \\
\mathrm{si}>70 \text { años }\end{array}$ & $\begin{array}{l}\text { Déficit leve a moderado } \\
\text { (por métodos de evaluación } \\
\text { validados) }\end{array}$ \\
\hline $\begin{array}{l}\text { Grado } 2 \text { Malnutrición Severa } \\
\text { (Requiere un criterio fenotípico que } \\
\text { cumpla este grado) }\end{array}$ & $\begin{array}{l}>10 \% \text { en los últimos } 6 \text { meses, }> \\
20 \% \text { arriba de } 6 \text { meses }\end{array}$ & $\begin{array}{l}<18,5 \mathrm{si}>70 \text { años o }<20 \\
\mathrm{si}>70 \text { años }\end{array}$ & $\begin{array}{l}\text { Déficit severo (por métodos de } \\
\text { evaluación validados) }\end{array}$ \\
\hline
\end{tabular}

IMC: Índice de masa muscular.

ello sigue siendo una prueba de utilidad en el diagnóstico de malnutrición.

A la fecha solo los criterios ASPEN/AND han sido validados. Un reciente estudio sobre la base de 5.606 pacientes encontró que aquellos pacientes clasificados como malnutridos severos, según estos criterios, tuvieron mayor estancia hospitalaria así como mortalidad que aquellos que no lo estaban. En una submuestra de este mismo estudio se reportó una sensibilidad de 96,1 $\%$ y una especificidad de 99,0 \% para identificar la malnutrición $^{(33)}$. Es necesario que los criterios GLIM sean evaluados y validados con estudios de calidad para así confirmar su utilidad en la práctica clínica.

\section{CONCLUSIONES}

La presencia de inflamación ha sido reconocida en la etiología de la malnutrición hospitalaria y en los criterios para su diagnóstico y clasificación. Estos criterios han sido propuestos por las principales sociedades de nutrición parenteral y enteral en el ámbito mundial y están actualmente en proceso de validación.

En presencia de inflamación los valores de albúmina y prealbúmina no deberán ser interpretados desde un enfoque nutricional, pero sí como potentes marcadores de la severidad de la injuria, el pronóstico y las alteraciones del metabolismo. De manera que cuanto más bajos los valores, mayor será el grado de injuria, complicaciones asociadas, y peor pronóstico, y por consiguiente la intervención nutricional se convierte en una prioridad.

Durante la estancia hospitalaria se deberá detectar y diagnosticar aquellos pacientes malnutridos e iniciar un oportuno plan de intervención nutricional. Sin embargo, la recuperación nutricional deberá continuar aun después de que el paciente sea dado de alta.

\section{Financiamiento}

El presente artículo no tuvo financiación.

\section{Conflicto de intereses}

El autor declara no tener conflicto de intereses.

\section{Referencias bibliográficas}

1. UNICEF. Facts for life. [Internet]. Unicef; 2010. [consultado el 1 de Julio 2019]. Disponible en: https://www.unicef.org/ publications/index_53254.html

2. Organización Mundial de la Salud. Nutrición. Desafíos. [Internet]. WHO, 2019. [consultado el 1 de Julio 2019]. Disponible en: https://www.who.int/nutrition/challenges/es/

3. Butterworth CE Jr. The skeleton in the hospital closet. Nutrition. 1994; 10(5):442.

4. Sauer AC, Goates S, Malone A, Mogensen KM, Gewirtz G, Sulz I, et al. Prevalence of Malnutrition Risk and the Impact of Nutrition Risk on Hospital Outcomes: Results From nutritionDay in the US. JPEN J Parenter Enteral Nutr. 2019. doi: 10.1002/jpen.1499

5. Soeters PB, Reijven PL, van Bokhorst-de van der Schueren MA, Schols JM, Halfens RJ, Meijers JM, et al. A rational approach to nutritional assessment. Clin Nutr. 2008;27(5):706-16.

6. Braunschweig C, Gomez S, Sheean PM. Impact of declines in nutritional status on outcomes in adult patients hospitalized for more than 7 days. J Am Diet Assoc. 2000; 100(11):1316-22.

7. Mechanick JI. Practical aspects of nutritional support for wound-healing patients. Am J Surg. 2004; 188(1A Suppl): 52-6.

8. Kubrak C, Jensen L. Malnutrition in acute care patients: a narrative review. Int J Nurs Stud. 2007;44(6):1036-54.

9. Gout BS, Barker LA, Crowe TC. Malnutrition identification, diagnosis and dietetic referrals: are we doing a good enough job? Nutr Diet. 2009; 66(4):206-11.

10. Cerra FB, Benitez MR, Blackburn GL, Irwin RS, Jeejeebhoy $\mathrm{K}$, Katz DP, RR. Applied nutrition in ICU patients. A consen- 
sus statement of the American College of Chest Physicians. Chest. 1997;111(3):769-78.

11. Krishnan JA, Parce PB, Martinez A, Diette GB, Brower RG. Caloric intake in medical ICU patients: consistency of care with guidelines and relationship to clinical outcomes. Chest. 2003;124(1): 297-305.

12. Doig GS, Simpson F, Bellomo R, Heighes PT, Sweetman EA, Chesher D, et al. Intravenous amino acid therapy for kidney function in critically ill patients: a randomized controlled trial. Intensive care medicine. Intensive Care Med. 2015; 41(7): 1197-208.

13. Ishibashi N, Plank LD, Sando K, Hill GL. Optimal protein requirements during the first 2 weeks after the onset of critical illness. Crit Care Med. 1998; 26(9): 1529-35.

14. Plank LD. Protein for the critically ill patient-what and when? Eur J Clin Nutr. 2013; 67(5): 565.

15. Muscaritoli M, Anker SD, Argiles J, Aversa Z, Bauer JM, Biolo $\mathrm{G}$, et al. Consensus definition of sarcopenia, cachexia and pre-cachexia: joint document elaborated by Special Interest Groups (SIG) "cachexia-anorexia in chronic wasting diseases" and "nutrition in geriatrics". Clin Nutr. 2010; 29(2):154-9.

16. Arends J, Baracos V, Bertz H, Bozzetti F, Calder PC, Deutz NE, et al. ESPEN expert group recommendations for action against cancer-related malnutrition. Clin Nutr. 2017; 36(5): 1187-96.

17. Beutler B, Cerami A. Tumor necrosis, cachexia, shock, and inflammation: a common mediator. Ann Rev Biochem. 1988; 57:505-18.

18. Morley JE, Thomas DR, Wilson MM. Cachexia: pathophysiology and clinical relevance. Am J Clin Nutr. 2006; 83(4):735-43.

19. Jensen GL. Inflammation as the key interface of the medical and nutrition universes: a provocative examination of the future of clinical nutrition and medicine. Journal of Parenteral and Enteral Nutrition. 2006; 30(5):453-63.

20. Banh L. Serum proteins as markers of nutrition: what are we treating? Pract gastroenterol. 2006, 30(10):46-64.

21. Nicholson JP, Wolmarans MR, Park GR. The role of albumin in critical illness. Br J Anaesth. 2000; 85(4): 599-610.

22. Stosovic MD, Naumovic RT, Stanojevic ML, Simic-Ogrizovic SP, Jovanovic DB, Djukanovic LD. Could the level of serum albumin be a method for assessing malnutrition in hemodialysis patients? Nutr Clin Pract. 2011; 26(5):607-13.

23. Mendez CM, McClain CJ, Marsano LS. Albumin therapy in clinical practice. Nutr Clin Pract. 2005; 20(3):314-20.
24. Tayek JA. Albumin synthesis and nutritional assessment. Nutr Clin Pract. 1988; 3(6): 219-21.

25. White JV, Guenter P, Jensen G, Malone A, Schofield M, Academy Malnutrition Work Group, ASPEN Malnutrition Task Force, ASPEN Board of Directors. Consensus statement: Academy of Nutrition and Dietetics and American Society for Parenteral and Enteral Nutrition: characteristics recommended for the identification and documentation of adult malnutrition (undernutrition). JPEN J Parenter Enteral Nutr. 2012; 36(3): 275-83.

26. Jensen GL, Mirtallo J, Compher C, Dhaliwal R, Forbes A, Grijalba RF, et al. Adult starvation and disease-related malnutrition: a proposal for etiology-based diagnosis in the clinical practice setting from the International Consensus Guideline Committee. JPEN J Parenter Enteral Nutr. 2010; 34(2):156-9.

27. Cederholm T, Barazzoni R, Austin P, Ballmer P, Biolo G, Bischoff SC, et al. ESPEN guidelines on definitions and terminology of clinical nutrition. Clinical Nutrition. 2017;36(1):49-64.

28. Cederholm T, Jensen GL, Correia MI, Gonzalez MC, Fukushima R, Higashiguchi T, et al. GLIM criteria for the diagnosis of malnutrition-A consensus report from the global clinical nutrition community. Clin Nutr. 2019; 38(1): 1-9.

29. Norman K, Stobäus N, Gonzalez MC, Schulzke JD, Pirlich M. Hand grip strength: outcome predictor and marker of nutritional status. Clin Nutr. 2011; 30(2): 135-42.

30. Windsor JA, Hill GL. Grip strength: a measure of the proportion of protein loss in surgical patients. Br J Surg. 1988; 75(9): 880-2.

31. Peng S, Plank LD, McCall JL, Gillanders LK, McIlroy K, Gane EJ. Body composition, muscle function, and energy expenditure in patients with liver cirrhosis: a comprehensive study. American J Clin Nutr. 2007; 85(5): 1257-66.

32. Vaz M, Thangam S, Prabhu A, Shetty PS. Maximal voluntary contraction as a functional indicator of adult chronic undernutrition. Br J Nutr. 1996; 76(1): 9-15.

33. Hiura G, Lebwohl B, Seres DS. Malnutrition Diagnosis in Critically Ill Patients Using 2012 Academy of Nutrition and Dietetics/American Society for Parenteral and Enteral Nutrition Standardized Diagnostic Characteristics Is Associated With Longer Hospital and Intensive Care Unit Length of Stay and Increased In-Hospital Mortality. JPEN J Parenter Enteral Nutr. 2019. doi: 10.1002/jpen.1599. 